\title{
The Role of Rat Medial Frontal Cortex in Effort-Based Decision Making
}

\author{
Mark E. Walton, David M. Bannerman, and Matthew F. S. Rushworth \\ Department of Experimental Psychology, Oxford, OX1 3UD, United Kingdom
}

We conducted a series of experiments to elucidate the role of rat medial frontal cortex (MFC) (including prelimbic, infralimbic, and cingulate cortices) in effort-based decision making. Rats were trained on a cost-benefit T-maze task in which they could either choose to climb a barrier to obtain a high reward in one arm (HR arm) or could obtain a small reward in the other with no barrier present (LR arm). Before surgery, all animals were selecting the HR arm on almost every trial. However, after excitotoxic lesions to MFC, the rats shifted to selecting the LR arm on almost every trial. This was not caused by a spatial memory or motor deficit because the same rats returned to selecting the HR arm when the amount of effort needed to be expended to obtain reward in either arm was equalized by putting an iden- tical barrier in the LR arm. Additional experiments demonstrated that the change in effort-based decisions observed in the rats was not caused by a complete insensitivity to reward and effort because they returned to choosing the HR arm if either the cost was reduced (by making the barrier smaller) or the benefit was increased (increasing the food ratio differential). Instead, the MFC lesion shifted the animals' decision criterion, making them more likely to choose the LR arm than the shamlesioned animals. These results imply that medial frontal cortex is important for allowing the animal to put in more work to obtain greater rewards.

Key words: decision making; cost-benefit; effort; medial frontal cortex; prelimbic cortex; cingulate
The nucleus accumbens (NAc) has been implicated in making decisions about costs and benefits of different courses of action (Salamone et al., 1997; Cardinal et al., 2001). Dopamine depletions in this region biased animals to prefer to exert less behavioral effort for a smaller or less palatable reward (Neill and Justice, 1981; Cousins and Salamone, 1994; Salamone et al., 1994; Cousins et al., 1996). Similarly, excitotoxic NAc lesions caused rats to shift from preferring to wait for larger reward to choosing small immediate reward (Cardinal et al., 2001). NAc is only one part of an interconnected frontostriatal network of regions and receives extensive projections from medial frontal cortex (MFC) in the rat (Berendse et al., 1992; Brog et al., 1993). A similar circuit appears present in primates (Haber et al., 1995), although there is debate as to the exact anatomical homologies between the different species (Uylings and van Eden, 1990; Conde et al., 1995; Preuss, 1995).

There are several reasons to believe that MFC might also be important in motivating cost-benefit decisions. Feeding and response-contingent food rewards are both associated with changes in extracellular dopamine in MFC (Feenstra and Botterblom, 1996; Bassareo and Di Chiara, 1997; Richardson and Gratton, 1998), and single-unit recording has shown cell responses in this region that differentiate between high and low reward sizes (Jung et al., 1998; Pratt and Mizumori, 2001). Moreover, MFC lesions disturb normal feeding behavior (Kolb, 1984)

Received July 11, 2002; revised Oct. 2, 2002; accepted Oct. 3, 2002

This work was funded by the Medical Research Council and a Wellcome Prize Studentship (M.E.W.). D.M.B. was funded from a Wellcome Project grant awarded to Prof. J. N. P. Rawlins. We thank Nick Rawlins and John Salamone for helpful advice about experimental protocol and Greg Daubney and Caroline Healy-Yorke for histology.

Correspondence should be addressed to Mark Walton, David Bannerman, or Matthew Rushworth, Department of Experimental Psychology, South Parks Road, Oxford, OX1 3UD, UK. Email: mark.walton@psy.ox.ac.uk, david. bannerman@psy.ox.ac.uk, or matthew.rushworth@psy.ox.ac.uk.

Copyright (C) 2002 Society for Neuroscience 0270-6474/02/2210996-08 $\$ 15.00 / 0$ and reduce food hoarding (de Brabander et al., 1991; Lacroix et al., 1998). However, it is uncertain what, if any, role MFC plays in cost-benefit decisions. Human neuroimaging studies show activity in medial frontal and anterior cingulate cortices, as well as NAc, when making risk-based choices (Elliott and Dolan, 1998; Rogers et al., 1999). Nevertheless, the one study to address this issue directly found no effect of MFC lesions on rats' response selection when the cost was in terms of delay in reward delivery (Cardinal et al., 2001). One possibility is that different circuits might subserve different types of cost-benefit decision depending on what factor is acting as the cost. Several of the behaviors that are impaired after MFC lesions (such as food hoarding) involve working to receive reward, and frontostriatal circuits have been described as translating motivation into action (Mogenson et al., 1980; Paus, 2001).

We therefore returned to a T-maze cost-benefit procedure originally developed by Salamone et al. (1994) to investigate the role of rat MFC in choosing how much effort to exert to obtain larger rewards. Rats could choose to either obtain small rewards in one arm or climb a barrier in the other to acquire large rewards. Medial frontal lesions were made to include prelimbic (PL), infralimbic (IL), and cingulate (CG) regions, all of which project to NAc, as one possible reason for the difficulty in finding consistent deficits after lesions to MFC has been the difference in type and extent of the cell loss (Aggleton et al., 1995; Dias and Aggleton, 2000).

\section{MATERIALS AND METHODS}

Animals. Twenty-four male Lister hooded rats (Harlan Olac, Bicester, UK) were used for this study. They were $\sim 2$ months old at the start of training and had never been used for any other experiment. All were housed in groups of three in a room with a $12 \mathrm{hr}$ light/dark cycle (lights on at 7:00 A.M., with testing always occurring during the light phase of the day). During the experiment, the animals were maintained on a restricted diet at $\sim 85 \%$ of their free-feeding weight but had access to water ad libitum in their home cages at all times. The experiments 

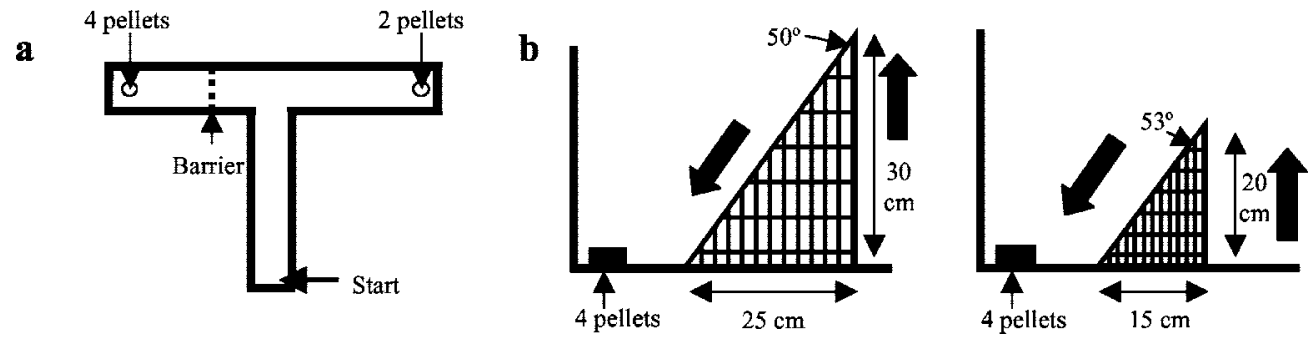

Figure 1. $a$, Schematic top view of the high-walled T-maze apparatus used throughout the test phase of the study. On each choice trial, the animals could either choose the LR arm with two pellets or could climb over a wire mesh barrier to obtain four pellets in the HR arm. The HR arm was to the left for half of the animals and to the right for the others. $b$, The barriers were constructed out of thick wire mesh in the shape of a right-angled triangle. The rats climbed up the vertical side and descended down the slope to obtain the HR reward. In experiments 1 , 2, and 4, the barrier was always $30 \mathrm{~cm}$. In experiment 3, the barrier was either $30 \mathrm{~cm}$ (left) or $20 \mathrm{~cm}$ (right), depending on the condition.

described were conducted in accordance with the United Kingdom Animals Scientific Procedures Act (1986), under project license number PPL 30/1505.

Apparatus. The rats were tested on an elevated, high-sided wooden T-maze, placed $80 \mathrm{~cm}$ above floor level (Fig. 1a). The start arm joined on to two goal arms, all of which were $60 \mathrm{~cm}$ in length, $10 \mathrm{~cm}$ wide, and had walls of $30 \mathrm{~cm}$ in height. A raised metal food well $(3 \mathrm{~cm}$ in diameter) was situated at the far end of each goal arm, $2 \mathrm{~cm}$ from the back wall. On "forced" trials, a $30 \mathrm{~cm}$ tall and $10 \mathrm{~cm}$ wide wooden block was used to prevent access to one of the goal arms. The maze was painted a uniform gray color throughout.

The barriers over which the animals had to climb to obtain rewards were constructed out of wire mesh in the shape of a three-dimensional right-angled triangle. This meant that the animals had to scale the vertical side but were able to descend down a slope of varying incline (depending on the size of barrier). There were two climbing barriers, each with two different length sides, resulting in a total of four sizes of barrier $(15,20,25$, and $30 \mathrm{~cm}$ ) (Fig. $1 b$ ).

Habituation and training. Before the start of training, the rats were handled every day for 1 week by the experimenter to familiarize them with human contact and were put on a restricted feeding schedule. When they reached $85 \%$ of their free-feeding weight, the rats were introduced to the T-maze. On the first day, the animals were placed in the start arm in cages of three and were allowed to explore the maze for $10 \mathrm{~min}$. Plentiful food was left in both feeding wells in the goal arms (45 mg food-reinforcement pellets, Formula A/I; P. J. Noyes, Lancaster, NH). The second day of habituation was identical except for the fact that each animal investigated the maze individually. At the end of these $2 \mathrm{~d}$, all of the rats were eating the pellets in the food wells.

The first phase of discrimination training involved putting four pellets in the feeding well of one goal arm [the high food arm (HR)] and two pellets in the other goal arm [low food arm (LR)]. For half of the rats, the HR arm was to the left, and, for the others, it was to the right. Initially, each rat was placed in the start arm and was allowed to sample both food arms on each trial. The rats were cycled in groups of six, meaning that there was an intertrial interval of $\sim 5 \mathrm{~min}$. There were five trials run each day over $2 \mathrm{~d}$. For the next $2 \mathrm{~d}$, the rats were moved onto the second phase in which access to one of the goal arms was prevented by placing a wooden block at its entrance (forced trials), thus forcing the rat to sample a particular arm on each trial. The order of the forced trials was determined pseudorandomly so that they never had more than two consecutive turns to either side. Each animal ran 10 trials per day. The final phase was similar to the first one (i.e., free choice) except that the rats were removed from the maze after eating the food in the selected arm without being able to sample from the other arm. On trials 6 and 12, the block was placed in the arm chosen by the rat on its previous trial to try to prevent turn biases interfering with discrimination training (e.g., if the rat had selected the left arm on trial 5, then it would be forced to go right on trial 6, regardless of the location of the HR and LR arms).

When all of the rats were choosing to collect the four pellets on $>90 \%$ of occasions during the training session, the $15 \mathrm{~cm}$ barrier was placed in the center of the HR arm. For the first five trials, the animals were only removed from the maze once they had climbed the barrier and eaten the four food pellets. Thereafter, and for all subsequent training, trials were run on a choice basis, meaning that the rats would be returned to the holding cage immediately after consuming the food in the selected arm. The barrier size was increased by $5 \mathrm{~cm}$ every $3 \mathrm{~d}$ up to a maximum of 30 $\mathrm{cm}$, and each rat ran 10 trials per day.

Surgery. Rats received excitotoxic bilateral MFC lesions $(n=12)$ or sham surgery $(n=12)$. Assignment of animals to groups was counterbalanced with respect to preoperative performance and the right-left orientation of the rewards. All animals were anesthetized with Avertin $(0.29 \mathrm{gm} / \mathrm{kg}$, i.p. $)$ and placed in a stereotaxic frame with the head level between bregma and lambda. An incision was made along the midline, and a dental drill was used to remove a portion of bone overlying the injection sites. Injections were made with a $10 \mu \mathrm{l}$ syringe with a specially adapted 34 gauge needle mounted onto the stereotaxic frame. The lesion was produced by infusing $0.5 \mu \mathrm{l}$ of quinolinic acid $(0.09 \mathrm{M})$ at the following anteroposterior (AP), mediolateral (ML), and dorsoventral (DV, from brain surface) coordinates relative to bregma: AP +3.0, ML $\pm 0.5, \mathrm{DV}-1.5$ and $-3.0 ; \mathrm{AP}+2.3, \mathrm{ML} \pm 0.5, \mathrm{DV}-2.0 ; \mathrm{AP}+1.6, \mathrm{ML}$ $\pm 0.5, \mathrm{DV}-2.0 ; \mathrm{AP}+0.9, \mathrm{ML} \pm 0.5, \mathrm{DV}-2.0$; and $\mathrm{AP}+0.2, \mathrm{ML} \pm 0.5$, DV -2.0 . These coordinates were based on pilot lesions and on the work of Robbins and colleagues on medial frontal and anterior cingulate cortex (Bussey et al., 1997a,b). Infusions were made manually at a rate of $0.1 \mu \mathrm{l}$ every $30 \mathrm{sec}$ with a $30 \mathrm{sec}$ interval between injections. On completion of each infusion, the needle was left in place for another 3 min to ensure that diffusion occurred away from the injection site. Sham lesions were produced in the same way, except that the needle was not lowered into the cortex and no neurotoxin was injected. On completion of surgery, all animals were sutured, and a topical antibiotic powder (P.E.P. 2\% powder; Intervet Laboratories, Milton Keynes, UK) was sprinkled over the wound.

Experiment 1. The testing protocol for all of the experiments was identical, with the only changes between them being in the size or quantity of barriers or in the ratio of the food reward. The first two trials of any day were forced in opposite directions, meaning that the animals had to sample both arms before receiving any test trials. Forced trials were counterbalanced across animals and across days. Each animal then ran 10 choice trials per day for a period of $3 \mathrm{~d}$ (this $3 \mathrm{~d}$ period is referred to as a "testing block"), except in the case in which a stable baseline was required, in which case the animals were tested until the group scores were within $\pm 4 \%$ for 3 consecutive days. A schematic diagram of the different stages of testing can be observed in Figure 2. Each testing block has been assigned a letter for ease of referral.

For experiment 1 , the $30 \mathrm{~cm}$ barrier was placed in the $\mathrm{HR}$ arm and the LR arm was empty. Two prelesion testing blocks were run with a break of 6 weeks in between (Fig. 2, testing blocks $A, B$ ). After a 2 week recovery period after surgery, all animals were put back on a restricted diet. Testing began again $3 \mathrm{~d}$ later (after they returned to $85 \%$ of their free-feeding weight). The single postlesion testing block was conducted in the same manner as the prelesion testing (block C).

Experiment 2. To assess whether any deficit represented a simple motor or spatial impairment, or an inability to process reward quantity information, an identical $30 \mathrm{~cm}$ barrier was placed in the LR arm. This meant that the rats had to climb over a barrier to obtain any reward and that the effort expended on each trial was the same, regardless of which goal arm was chosen (block D). Afterward, the rats were retested with just the single barrier in the HR arm (block E).

Experiment 3. After the retest testing block in experiment 2, the rats continued to run with the barrier in the HR arm until a stable baseline was reached (block F). The $30 \mathrm{~cm}$ barrier was then replaced with a $20 \mathrm{~cm}$ one to determine whether either group would alter their behavior with a reduction in the effort needed to obtain the four pellets (block $G$ ). The animals were tested as before. However, to ascertain whether the rats' choices were genuinely different from the case in which effort was equalized between the goal arms, they were run again as in experiment 2 with a $30 \mathrm{~cm}$ barrier in each arm (block $\mathrm{H}$ ) before finally being retested with the single $20 \mathrm{~cm}$ barrier (block I).

Experiment 4. In this experiment, we wanted to see whether increasing the reward ratio differential would have similar effects as decreasing the effort needed to obtain the reward. The rats were returned to the single 


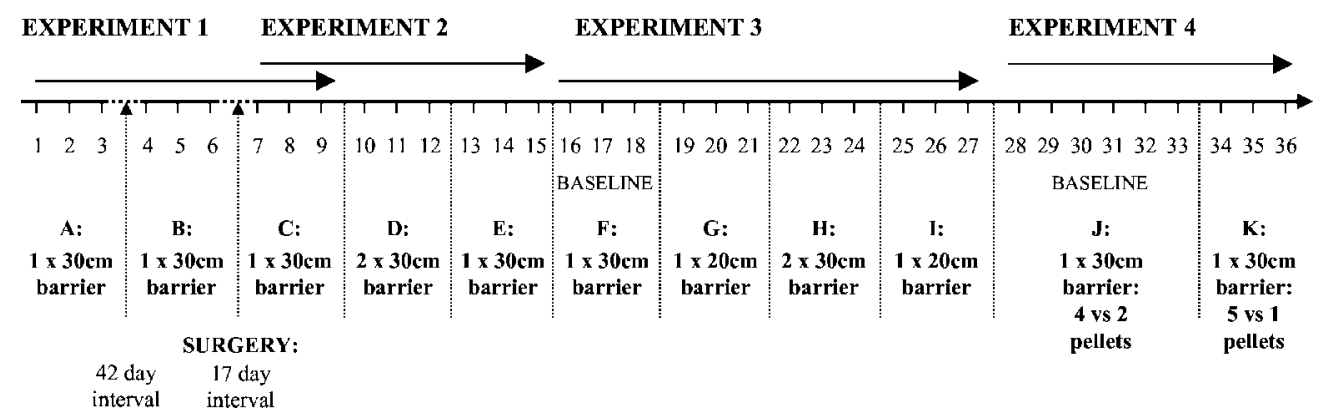

$30 \mathrm{~cm}$ barrier paradigm until a baseline level of performance was reached (block J). The ratio of reward sizes was then altered so that, instead of there being four pellets in the HR arm and two in the LR one, there were five and one pellets in the HR and LR arms, respectively (block K). Otherwise, the testing procedure was identical to that in experiment 1.

Histology. At the conclusion of behavioral testing, the rats were deeply anesthetized with Euthatal $(200 \mathrm{mg} / \mathrm{kg})$ and perfused transcardially with physiological saline and $10 \%$ Formalin saline. The brains were then removed and placed into a Formalin saline solution. Subsequently, the brains were placed in a sucrose-formalin solution for $24 \mathrm{hr}$, frozen, and then sectioned coronally $(50 \mu \mathrm{m})$. All sections were mounted and stained with cresyl violet. Histological evaluation was conducted by an experimenter who was unaware of the rats' behavioral performance. The lesions are described in terms of the nomenclature and classification of cortical areas adopted by Paxinos and Watson (1998).

\section{RESULTS}

\section{Histology}

The lesions of the medial frontal cortex were highly reproducible and, in most cases, destroyed all of PL-IL and CG cortices as intended (Figs. 3, 4). In the majority of the animals, there was some additional damage to orbital, secondary motor, and dorsopeduncular cortices. The transition between fully healthy cells and areas in which there was total cell loss was gradual. This resulted in a penumbral region in which some cells were present but those that remained were abnormal compared with the healthy cells observed in sections taken from sham-operated rats (Figs. 3, stippled area, 4). The presence of holes in the tissue after excitotoxic lesions (Fig. 4, middle row, left panel) is well documented with long survival times (4 months in this study). These do not indicate mechanical damage or section of axons en passage (Jarrard, 1989).

In most cases, cell loss was evident in the most anterior sections of the frontal lobe (5.2 $\mathrm{mm}$ anterior to bregma). In fact, only in the very smallest lesions (Fig. 3, left column) was there sparing at this level. At this point, when present, the lesion encompassed parts of orbitofrontal cortex (although there was sparing of tissue in most animals, especially of ventral orbital cortex) and encroached slightly into frontal association cortex. The lesion then completely removed PL-IL in all animals, except for the one with the smallest lesion (Fig. 3, left column). In addition, at this level, there was significant cell loss in secondary motor cortex and small but consistent damage to the dorsopeduncular cortex ventral to PL-IL. The lesion produced complete cell loss in the cingulate cortex in all animals, extending to a point $\sim 1 \mathrm{~mm}$ posterior to bregma (1.3 $\mathrm{mm}$ in the case of the largest lesion). In a small number of animals, there was also a small amount of damage to the medial edge of primary motor cortex at some levels dorsal to the corpus callosum. The damage to PL-IL and cingulate cortices was very consistent and near complete in all animals. Moreover, the observed behavioral deficits were present in all lesioned animals, regardless of the size of the damage.

In addition, in a small number of the animals, there appeared to be some thinning of the corpus callosum. This did not appear to be attributable to a direct effect of the neurotoxin because there was no evidence of direct mechanical damage into the fiber bundle. Furthermore, in all of the lesioned animals, there was some enlargement of the ventricles (Fig. 4 is representative), and, in a small number of the rats, there also appeared to be some shrinking of the dorsolateral component of the lateral septum. It is not clear as to whether this shrinking was attributable to distortion as a result of the ventricular enlargement or whether there was indeed some atrophy of the lateral septum. Again, it seems extremely unlikely that there was any direct neurotoxin-induced damage to the lateral septum because, as previously mentioned, the needle tracks always remained dorsal to the corpus callosum, and there was no evidence of direct mechanical damage to the fiber bundle. It is also unlikely that this damage had any overt behavioral consequences. There was, for example, no evidence of the septal hyper-reactivity syndrome in any of these rats (Fried, 1972).

\section{Experiment 1}

To analyze the results, the data were divided into separate $3 \mathrm{~d}$ testing blocks and were subjected to repeated-measures ANOVA with two within-subjects factors (day of testing, three; and testing block, two) and with group as the single between-subjects factor (sham vs lesion, two). All results are reported using the conservative Greenhouse-Geisser correction, which overcomes problems caused by violations of the sphericity assumption when using an $F$ test. Also, despite the fact that the ANOVA is an extremely robust procedure, in a few select cases, there is large nonhomogeneity of variance between the sham and lesion groups. Therefore, to verify the validity of our statistics, the data were inverted (to make the SD proportional to the mean), subjected to a logarithmic transformation, and reanalyzed. Any differences between this and the previous analysis of the untransformed raw data in which the largest variance on any one day of a testing block is more than four times the smallest (Howell, 1997) are reported in the text.

For experiment 1, the results were split into first and second prelesion testing blocks and the single postlesion block. As can be observed in Figure 5, both the lesion and sham groups climbed the barrier to obtain the four pellet reward more often than choosing the arm with two pellets before surgery. When the two prelesion testing blocks (A and B) were analyzed, there were no significant differences between the blocks $\left(F_{(1,22)}=0.43\right.$; NS) or groups $\left(F_{(1,22)}=0.12\right.$; NS) and no significant interaction between these factors $\left(F_{(1,22)}=0.43\right.$; NS). This indicates that the choices of both groups of rats were stable and consistent across a period of weeks. There was a significant effect of day $\left(F_{(1.33,29.35)}=4.29\right.$; $p<0.05)$ and a block $\times$ day interaction $\left(F_{(1.33,29.35)}=4.35 ; p<\right.$ $0.05)$ caused by fewer HR arm choices in both groups on day 1 of testing block 1 . 
5.2

4.2

3.2

2.2

1.6

0.7

$-0.2$

$-0.8$

$-1.3$

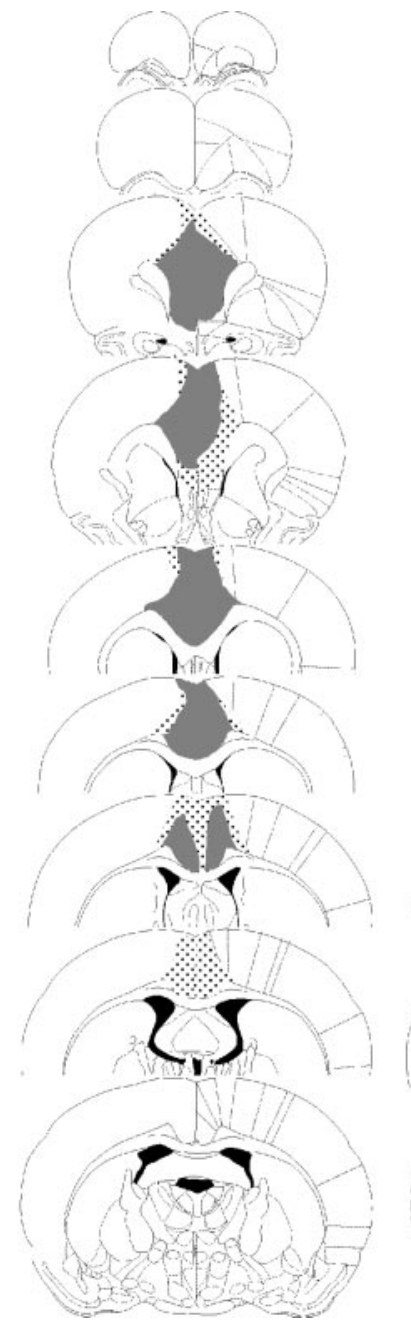

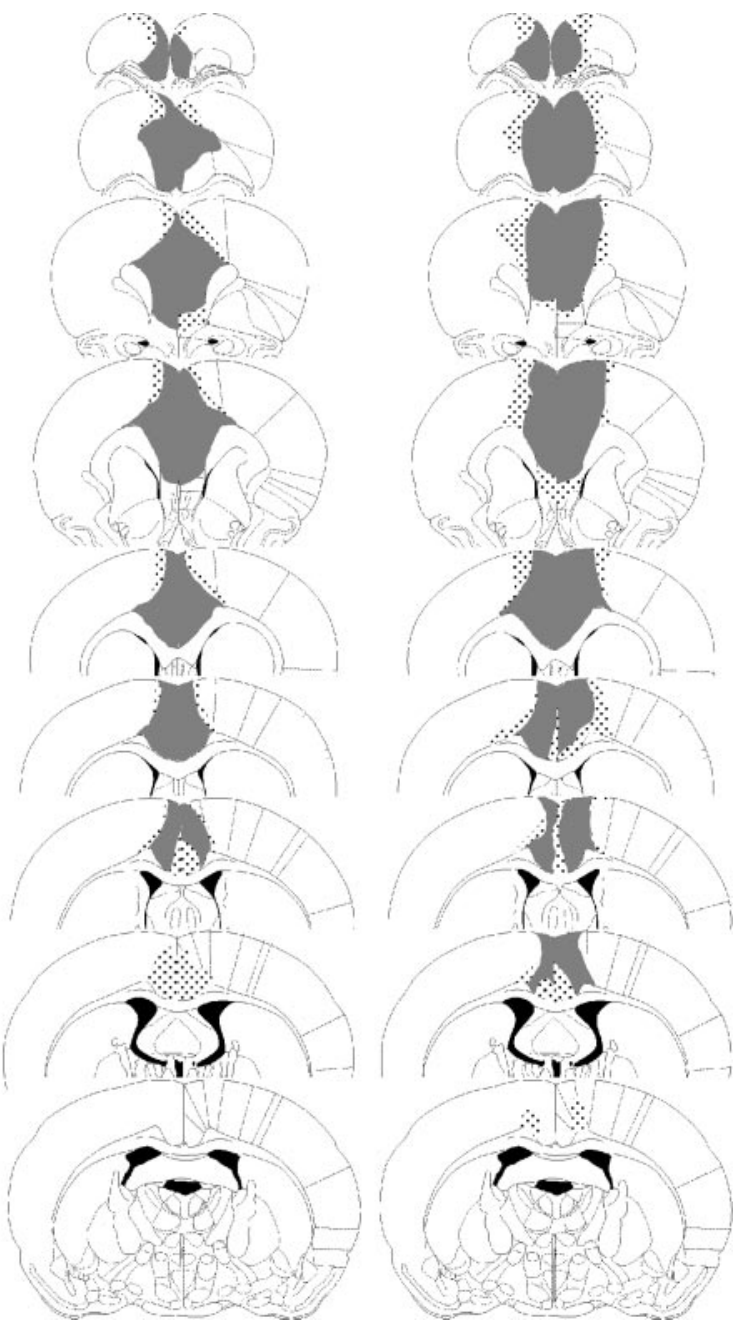

Figure 3. Reconstructions of medial frontal cortex lesions. Minimal (left column), representative (center column), and maximal (right column) extent of the lesions in coronal sections between $+5.2 \mathrm{~mm}$ anterior to bregma and -1.3 $\mathrm{mm}$ posterior to bregma are illustrated. Gray shading indicates areas of complete cell loss, whereas stippled shading represents the lesion penumbra in which cells were still present but clearly abnormal compared with those in shamoperated animals.
After surgery, the animals with medial frontal lesions showed a profound change in behavior and chose the LR arm on nearly every trial. In contrast, although they showed a slight drop in performance compared with before surgery, the sham group still responded to the HR arm on the vast majority of trials (Fig. 5). This was borne out by an ANOVA comparing the postlesion data (block C) with the second prelesion testing data (block B), which showed a significant interaction between block and group $\left(F_{(1,22)}=222.25 ; p<0.01\right)$, as well as main effects of these factors (block, $F_{(1,22)}=333.83, p<0.01$; group, $\left.F_{(1,22)}=52.82 ; p<0.01\right)$.

\section{Experiment 2: postlesion two-barrier test}

Results from experiment 2 are presented in the right side of Figure 5. The introduction of a second $30 \mathrm{~cm}$ barrier placed in the LR arm caused the lesion group to switch from choosing the LR arm on almost every trial to select instead the HR arm on the majority of trials. There was also a small shift in the behavior of the sham groups toward the HR arm. These results are illustrated by an ANOVA comparing the postlesion testing block (block $\mathrm{C}$ ) from experiment 1 with the data from the two-barrier testing block (block D), which revealed a significant interaction of testing block and group $\left(F_{(1,22)}=62.79 ; p<0.01\right)$, as well as individual main effects of the two factors (group, $F_{(1,22)}=163.67, p<0.01$; block, $\left.F_{(1,22)}=145.79, p<0.01\right)$. There was also a significant block $\times$ day $\left(F_{(1.89,41.52)}=16.68 ; p<0.01\right)$ and block $\times$ day $\times$ group $\left(F_{(1.90,41.77)}=14.01 ; p<0.01\right)$ interactions as a conse- quence of the fact that the percentage of trials on which the lesioned animals selected the HR arm increases sharply over the $3 \mathrm{~d}$ of testing. Although the main effect of day was present in the analysis of the raw data $\left(F_{(1.89,41.52)}=5.75 ; p<0.01\right)$, it did not reach significance after logarithmic transformation of the data $\left(F_{(1.86,40.92)}=2.89 ; p=0.07\right)$.

When the barrier was removed from the LR arm, the rats with medial frontal lesions returned over the 3 test days to choosing this arm on the majority of trials, whereas the sham group continued mainly to select the HR arm (Fig. 5). An ANOVA comparing this $3 \mathrm{~d}$ testing block (block E) with the two-barrier testing block (block D) once again revealed a block $\times$ day $\times$ group interaction $\left(F_{(1.81,39.84)}=16.61 ; p<0.01\right)$, as well as significant main effects of group $\left(F_{(1,22)}=36.71 ; p<0.01\right)$ and block $\left(F_{(1,22)}=60.48 ; p<0.01\right)$. Because the shift back to the LR arm was not immediate on its removal, there was also a significant block $\times$ day interaction $\left(F_{(1.81,39.84)}=16.85 ; p<0.01\right)$. However, the block $\times$ group interaction was only significant in the raw $\left(F_{(1,22)}=17.59 ; p<0.01\right)$ and not the transformed $\left(F_{(1,22)}=4.03\right.$; $p=0.06)$ data, so if does not appear to be robust.

\section{Experiment 3: $20 \mathrm{~cm}$ barrier test}

The animals were run with a single $30 \mathrm{~cm}$ barrier in the HR arm until their performance stabilized. However, in keeping with the analysis of previous experiments, only the last $3 \mathrm{~d}$ (Fig. 6, test days 16-18) of this period of testing have been used for comparison 
3.2
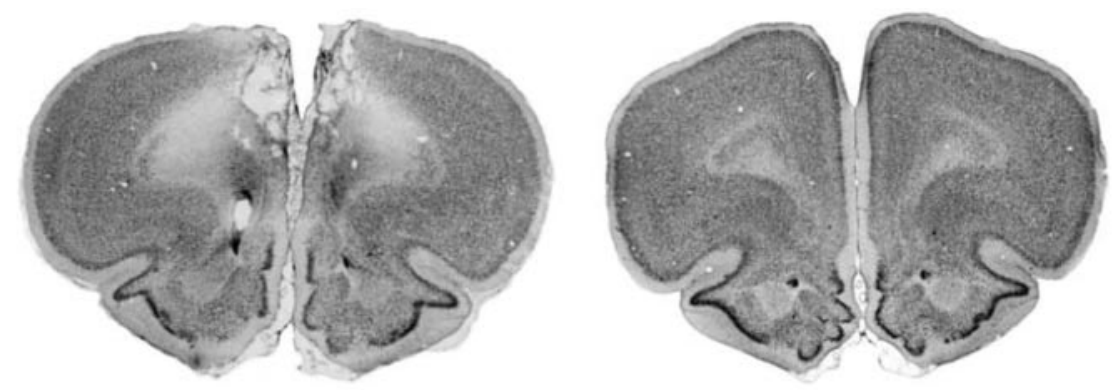

1.6
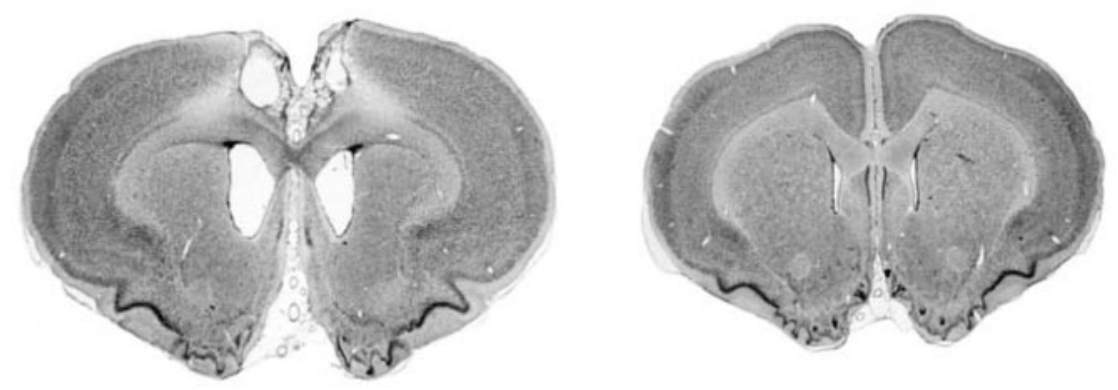

$-0.2$

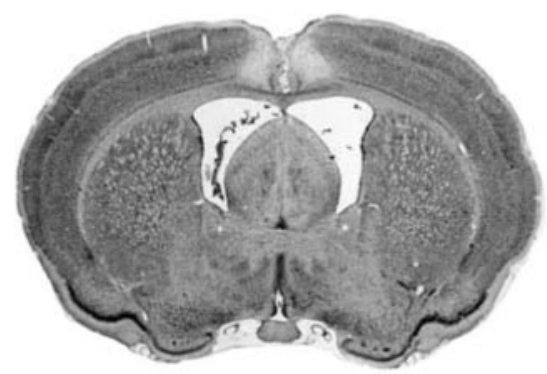

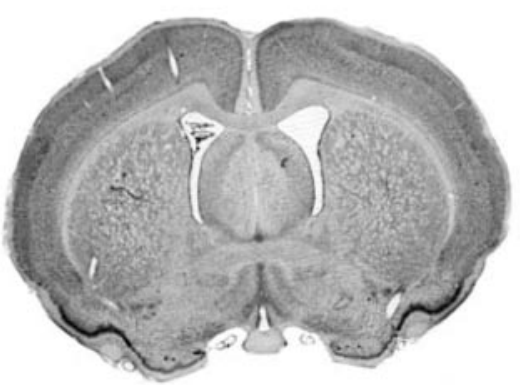

Figure 4. Photomicrographs of coronal sections showing typical cell loss in a representative lesioned rat (left column) compared with a sham-operated animal (right column). with the data obtained when the animals were subsequently tested with a single $20 \mathrm{~cm}$ barrier.

The replacement of the $30 \mathrm{~cm}$ barrier with a $20 \mathrm{~cm}$ one in the HR arm had the effect of making both groups of rats choose to obtain the four pellet reward more frequently (Fig. 6). This can be seen in an ANOVA comparing these two testing blocks (blocks F and $G$ ) in which, once again, there was a significant main effect block $\left(F_{(1,22)}=13.67 ; p<0.01\right)$. Nevertheless, there was no clear difference between the change in performance in the lesion and the sham groups because the block $\times$ day $\times$ group interaction only reached significance in the analysis of the raw data (raw data, $F_{(1.98,43.58)}=4.44, p<0.05$; transformed data, $F_{(1.97,43.34)}=2.26$, $p=0.12)$, and the block $\times$ group interaction failed to reach significance in either (raw data, $F_{(1,22)}=3.25, p>0.05$; transformed data, $\left.F_{(1,22)}=0.379, p=\mathrm{NS}\right)$. However, there was still an overall difference in the performance of the lesioned and sham animals (main effect of group, $F_{(1,22)}=36.18 ; p<0.01$ ). As in the previous experiment, this increase in responding to the HR arm happened over the course of the $3 \mathrm{~d}$ testing period, resulting in a significant main effect of day $\left(F_{(1.84,40.42)}=3.43 ; p<0.05\right)$ and significant block $\times$ day interaction $\left(F_{(1.98,43.58)}=5.12 ; p<0.05\right)$.

However, merely changing the height of the barrier did not have such a dramatic effect on the rats' decision as the addition of a second barrier did in experiment 2. An ANOVA comparing the performance of the $20 \mathrm{~cm}$ barrier testing block (block $\mathrm{G}$ ) with the subsequent testing block when the rats were retested with a $30 \mathrm{~cm}$ barrier in each arm (block $\mathrm{H}$ ) indicated a significant increase in the percentage of trials on which the HR arm was chosen in the two-barrier condition (block $\times$ group, $F_{(1,22)}=15.90, p<0.01$; block, $F_{(1,22)}=16.98, p<0.01$; group, $\left.F_{(1,22)}=21.63, p<0.01\right)$. Once again, there was a significant effect of day $\left(F_{(1.78,39.05)}=\right.$ $11.11 ; p<0.01)$ caused by the gradual shift in the lesioned animals' choices to the HR arm.

When the animals were retested with the single $20 \mathrm{~cm}$ barrier, the sham group continued to select the HR arm on almost every trial. The medial frontal animals, in contrast, shifted their behavior again, and there was a decrease in the percentage of trials on which they chose to climb the $20 \mathrm{~cm}$ barrier to obtain the four pellets that occurred over the $3 \mathrm{~d}$ of testing (blocks $\mathrm{H}$ and I). This resulted in a significant block $\times$ day $\times$ group interaction $\left(F_{(1.78,39.08)}=3.97, p<0.05\right.$; main effect of group, $F_{(1,22)}=12.00$, $p<0.01)$. However, the main effect of block was only significant in the analysis of the raw data (raw data, $F_{(1,22)}=4.60, p<0.05$; transformed data, $\left.F_{(2,44)}=2.60, p>0.05\right)$. The gradual shift in responding in the lesion group also meant that the block $\times$ group interaction just failed to reach significance $\left(F_{(1,22)}=4.00\right.$; $p=0.06)$.

\section{Experiment 4: five pellets versus one pellet}

As in experiment 3, all of the rats were then run on the original one $30 \mathrm{~cm}$ barrier paradigm until a stable baseline was achieved, and the final $3 \mathrm{~d}$ of this period of testing (Fig. 7, test days 31-33) were used in the subsequent ANOVA as a comparison with performance when the ratio of food pellets was changed from 4:2 


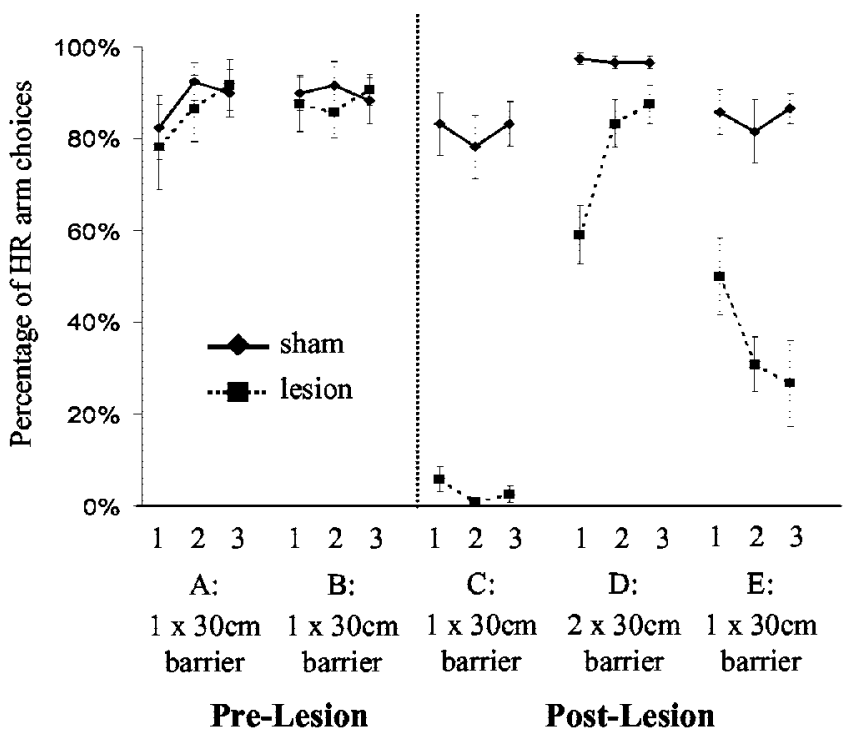

EXPERTMENT 1 EXPERIMENT 2

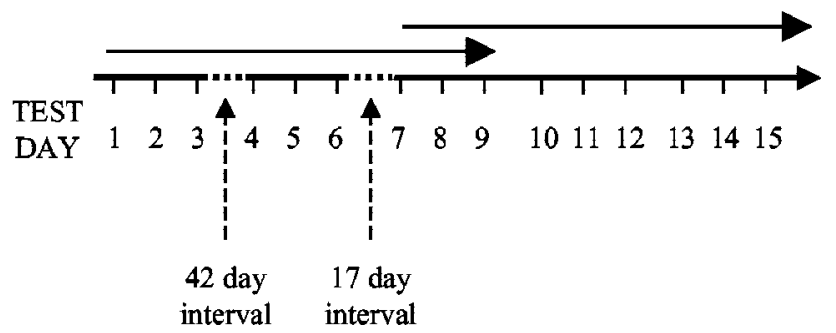

Figure 5. Mean \pm SE percentage of trials in which sham and lesion group rats chose the $\mathrm{HR}$ arm with a single $30 \mathrm{~cm}$ barrier in the HR arm. In experiment 1 , there was always just a single $30 \mathrm{~cm}$ barrier in the $\mathrm{HR}$ arm. However, in experiment 2, there could either be a single $30 \mathrm{~cm}$ barrier in the HR arm or identical $30 \mathrm{~cm}$ barriers in both goal arms. Each testing block consisted of $3 \mathrm{~d}$ of 10 choice trials per day.

to 5:1 (blocks $\mathrm{J}$ and $\mathrm{K}$ ). As can be observed in Figure 7, the increase in the differential of food pellets caused the lesion group to switch from selecting the LR arm on the majority of trials to choosing the HR arm, resulting in a significant interaction between block and group $\left(F_{(1,22)}=23.50, p<0.01\right.$; block, $F_{(1,22)}=$ $39.17, p<0.01$; group, $\left.F_{(1,22)}=17.54, p<0.01\right)$. There was also a main effect of day $\left(F_{(1.92,42.21)}=5.43 ; p<0.01\right)$, and this factor interacted significantly both with group $\left(F_{(1.92,42.21)}=5.94 ; p<\right.$ $0.01)$ and with testing block $\left(F_{(1.49,32.82)}=10.38 ; p<0.01\right)$ as a result of the fact that the number of trials on which the HR arm was selected by the lesion group increased markedly over the $3 \mathrm{~d}$ of testing with the 5:1 food ratio. By the final day of this test block, there was no difference in performance between the sham and lesion groups ( $t=1.20$; NS).

\section{DISCUSSION}

The results of experiment 1 and 2 demonstrate a profound change in effort-based decision making in rats with MFC lesions. Before surgery, the rats chose to climb the barrier to acquire the large reward on the vast majority of trials. However, after surgery, the lesion group switched their behavior and selected the LR arm on almost every trial. The size of this shift cannot be too strongly emphasized: over the $3 \mathrm{~d}$ of testing in experiment 1 , the lesioned animals chose the HR arm on only 11 trials of a total of 360 compared with 317 trials before surgery. This was true of all

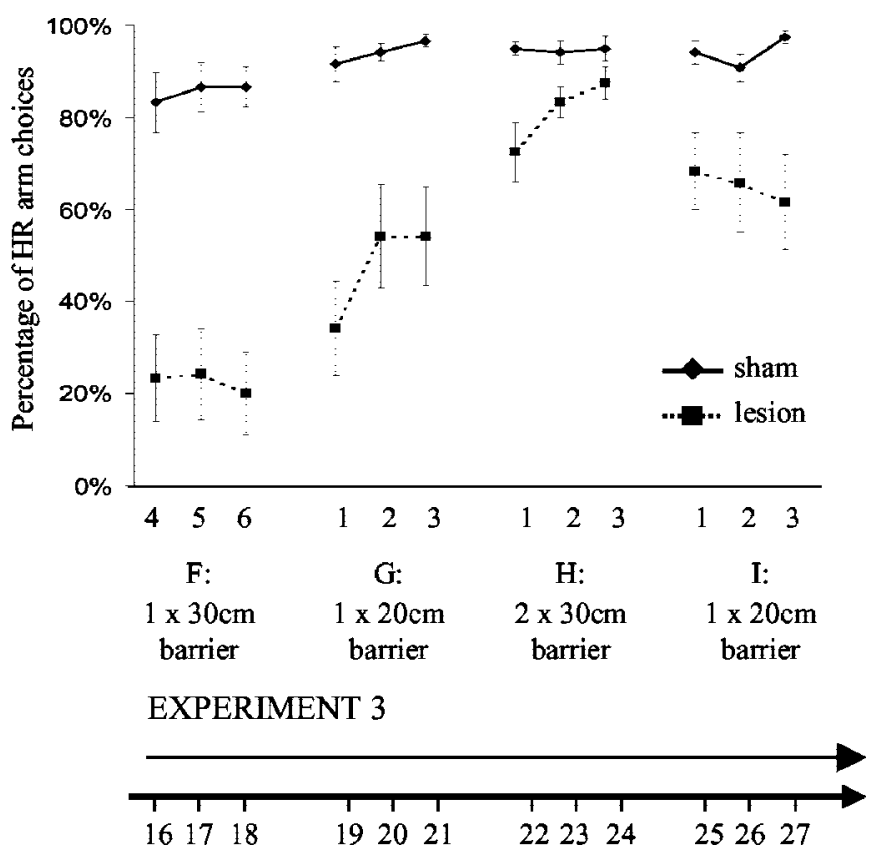

Figure 6. Mean $\pm \mathrm{SE}$ percentage of trials in which sham and lesion group rats chose the HR arm when the size of barrier was varied in the HR arm (experiment 3). Rats were run with either a single $20 \mathrm{~cm}$ barrier in the HR arm or with a $30 \mathrm{~cm}$ barrier in both goal arms. The first testing block (test days 16-18) is labeled 456 because it was a continuation of testing from the last $3 \mathrm{~d}$ of experiment 2 to get a consistent baseline measurement with the single $30 \mathrm{~cm}$ barrier before attempting any costbenefit manipulations.

lesioned animals, regardless of the amount of cell loss. Moreover, this change was stable across 4 weeks of continuous testing, with the lesion group showing only a small reduction in preference for the LR arm over the HR arm over the four experiments. Such a difference can neither be the result of the animals not remembering the respective reward quantities nor explained as a deficit in primary motivation or motor skills because the first two trials of each testing block were forced, meaning that all of the rats were required to sample both goal arms (including climbing the barrier in the HR arm) and consume food pellets before undergoing any choice trials. Moreover, the findings from experiment 2, which showed that, when the effort to obtain the food was equated between the two goal arms, the lesioned rats returned to climbing the barrier in the HR arm, rule out a complete insensitivity to reward and effort. Therefore, the pattern of responding can only be explained by a reduced preference to exert effort to acquire the large reward.

An important role for MFC in a reward-effort calculation is consistent with the study by Richardson and Gratton (1998) who recorded increases in dopamine signals in MFC when rats' response requirement was increased from a continuous fixed ratio 1 (FR1) schedule up to an FR3, FR5, or FR10 schedule. This is unlikely to be the result of changes in the rewarding properties of the reinforcer because a recent study showed that rats demonstrate no preferences for a particular food that they had to work hard to obtain in a previous study over one that was easily acquired (Armus, 2001). Instead, it implies that the amount of work required to earn reward impacts on MFC dopamine levels.

The results also complement those of Salamone and colleagues (Salamone et al., 1994; Cousins et al., 1996) who investigated the effects of dopamine depletions in NAc on a cost-benefit T-maze 


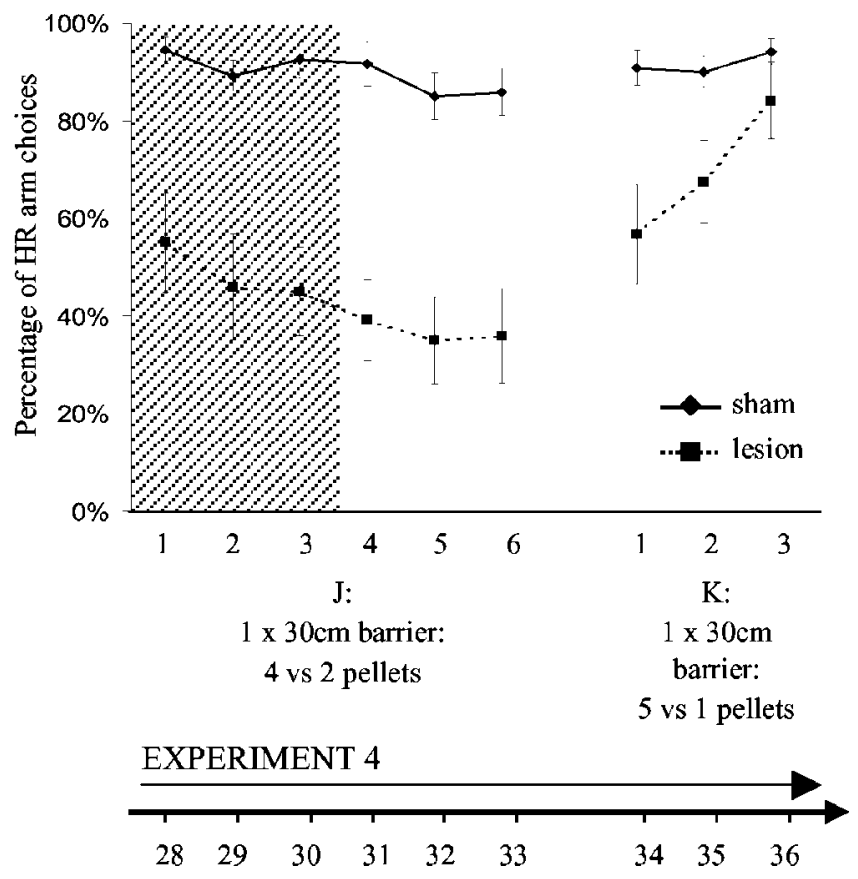

Figure 7. Mean \pm SE percentage of trials in which sham and lesion group rats chose the HR arm with a single $30 \mathrm{~cm}$ barrier in the $\mathrm{HR}$ arm in experiment 4 . The food differential ratio was changed from $4: 2$ in the baseline condition (HR/LR arm) to $5: 1$. The first $3 \mathrm{~d}$ of testing (test days 28-30; gray shading) when a stable baseline measure was being obtained have been included for the sake of completeness, although they have not been subjected to statistical analysis.

paradigm and found a similar, if less marked, shift in responding from the HR to the LR arm. They similarly concluded that their manipulation biased the animals' responses toward lower effort alternatives (Salamone et al., 1997). It is clearly an issue for additional research to investigate how MFC and NAc interact when choosing between actions on the basis of the amount of work needed to obtain rewards.

The fact that both MFC lesions and NAc dopamine depletions had similar effects on making decisions about effort can be understood in relation to the anatomy and physiology of the dopamine pathways in these regions. There are strong glutamatergic projections from all of the subregions of MFC to NAc (Sesack et al., 1989; Uylings and van Eden, 1990; Berendse et al., 1992; Brog et al., 1993), as well as indirect mechanisms by which MFC can influence NAc dopamine via the basal amygdala (Groenewegen et al., 1990; Jackson and Moghaddam, 2001). Moreover, it has been demonstrated that stimulation of MFC causes a selective increase in the firing of dopamine cells in the ventral tegmental area, the origin of the mesolimbic dopamine projections to NAc, and enhanced levels of dopamine in the NAc and MFC, whereas inhibition of MFC has the opposite effects (Murase et al., 1993; Taber and Fibiger, 1995; Jedema and Moghaddam, 1996).

However, the role of MFC in effort-based decisions demonstrated by the present study is in contrast to its apparent lack of function in delay-based impulsivity as measured by the paradigm by Cardinal et al. (2001). There are several plausible but not mutually exclusive reasons for this. First, in the study by Cardinal et al., complete MFC lesions were not made; instead, lesions of individual subregions were made separately in either PL-IL or $\mathrm{CG}$, both of which project directly to the core of NAc. It is therefore possible that the input from either one of these regions on its own was sufficient for rats to be able to avoid impulsive choices, whereas damage to both (as in the present study) would not be.

A second explanation is that distinct circuits or elements within the same frontal-accumbens circuit might be recruited differentially depending on the decision in hand. In many situations, an animal would have to balance the various costs and benefits of an action from several different sources. A response that requires more effort might also take more time and have more risks attached (such as foraging at a better food source farther afield). Our results might suggest that the MFC is only recruited in situations in which the amount of work needed to obtain reward becomes a crucial factor but not when time is of overriding importance (as in the study by Cardinal et al.). There is good evidence from the human neuroimaging literature on decision making that different regions are preferentially active depending on the level of risk or reward (Elliott and Dolan, 1998; Knutson et al., 2001; O'Doherty et al., 2001). In particular, the human anterior cingulate cortex seems to be activated by situations in which the outcome of actions can result in different sizes of reward (Bush et al., 2002; Gehring and Willoughby, 2002).

Although the results of experiment 1 showed a reversal of animals' decisions after a lesion to MFC, experiment 2 demonstrated that this was not caused by a complete insensitivity to costs and benefits. However, the first two experiments failed to indicate whether the rats had merely shifted their decision criterion, meaning that altering the cost-benefit ratio would cause them to modify their behavior as a consequence, or whether they always just take the easier option of the two. Experiments 3 and 4 convincingly showed the former to be more likely, as well as confirming our previous findings that MFC lesions do not make the rats totally insensitive to reward and effort. Both a reduction in the size of barrier from 30 to $20 \mathrm{~cm}$ (decreasing the cost) and increasing the food differential to 5:1 (increasing the benefit) caused the lesioned animals to change from choosing the LR arm to exerting effort to obtain the reward in the HR arm. The fact that performance with the $20 \mathrm{~cm}$ barrier was characterized by an increased preference to enter the $\mathrm{HR}$ arm than with the $30 \mathrm{~cm}$ barrier but reduced preference compared with the two-barrier condition shows that they were still able to weigh up relative costs and benefits in each new situation, even if their decision criterion appears to have been shifted markedly compared with the sham animals.

It is also of note, given that the ratio used in the study by Cardinal et al. (2001) was 4:1, that changing the food ratio to 5:1 in experiment 4 virtually equated the performance of the lesion and sham groups. This suggests that a third possible reason for the discrepancy between their findings and ours is their use of a single pellet as the low reward. Unfortunately, the experiments that have looked at the role of NAc in effort-based decisions did not systematically alter the cost-benefit ratio as was done in this study (Salamone et al., 1994; Cousins et al., 1996), so it is difficult to do more than speculate without additional data.

One noticeable aspect about the change in responding in the lesioned animals from the LR to HR arm and back again in all of the experiments was that it never occurred immediately. This was despite the fact that the rats were given experience of the addition and removal of the second barrier in the LR arm through the forced trials. One possibility is that this in part reflects an impairment in behavioral flexibility that several authors have suggested may occur after MFC lesions (Aggleton et al., 1995; Seamans et al., 1995; Ragozzino et al., 1999; Dias and Aggleton, 
2000). However, if any such deficit is present, it is secondary to the effect on the animals' decision making.

Taken as a whole, this study demonstrates a crucial role for rat MFC in effort-based decision making. However, rather than making the animals insensitive to rewards and effort, the effect of the lesion was to cause a shift in the decision criterion of the animals, meaning that they could still alter their behavior when the cost-benefit ratio was changed, although they showed a marked preference to choose the LR arm when compared with the controls. Whether the whole of MFC is uniquely involved in effort-based decision making will be the issue of additional research.

\section{REFERENCES}

Aggleton JP, Neave N, Nagle S, Sahgal A (1995) A comparison of the effects of medial prefrontal, cingulate cortex, and cingulum bundle lesions on tests of spatial memory: evidence of a double dissociation between frontal and cingulum bundle contributions. J Neurosci 15:7270-7281.

Armus HL (2001) Effect of response effort on the reward value of distinctively flavored food pellets. Psychol Rep 88:1031-1034.

Bassareo V, Di Chiara G (1997) Differential influence of associative and nonassociative learning mechanisms on the responsiveness of prefrontal and accumbal dopamine transmission to food stimuli in rats fed $a d$ libitum. J Neurosci 17:851-861.

Berendse HW, Galis-de Graaf Y, Groenewegen HJ (1992) Topographical organization and relationship with ventral striatal compartments of prefrontal corticostriatal projections in the rat. J Comp Neurol 316:314-347.

Brog JS, Salyapongse A, Deutch AY, Zahm DS (1993) The patterns of afferent innervation of the core and shell in the "accumbens" part of the rat ventral striatum: immunohistochemical detection of retrogradely transported fluoro-gold. J Comp Neurol 338:255-278.

Bush G, Vogt BA, Holmes J, Dale AM, Greve D, Jenike MA, Rosen BR (2002) Dorsal anterior cingulate cortex: A role in reward-based decision making. Proc Natl Acad Sci USA 99:523-528.

Bussey TJ, Everitt BJ, Robbins TW (1997a) Dissociable effects of cingulate and medial frontal cortex lesions on stimulus-reward learning using a novel Pavlovian autoshaping procedure for the rat: implications for the neurobiology of emotion. Behav Neurosci 111:908-919.

Bussey TJ, Muir JL, Everitt BJ, Robbins TW (1997b) Triple dissociation of anterior cingulate, posterior cingulate, and medial frontal cortices on visual discrimination tasks using a touchscreen testing procedure for the rat. Behav Neurosci 111:920-936.

Cardinal RN, Pennicott DR, Sugathapala CL, Robbins TW, Everitt BJ (2001) Impulsive choice induced in rats by lesions of the nucleus accumbens core. Science 292:2499-2501.

Conde F, Maire-Lepoivre E, Audinat E, Crepel F (1995) Afferent connections of the medial frontal cortex of the rat. II. Cortical and subcortical afferents. J Comp Neurol 352:567-593.

Cousins MS, Salamone JD (1994) Nucleus accumbens dopamine depletions in rats affect relative response allocation in a novel cost/benefit procedure. Pharmacol Biochem Behav 49:85-91.

Cousins MS, Atherton A, Turner L, Salamone JD (1996) Nucleus accumbens dopamine depletions alter relative response allocation in a T-maze cost/benefit task. Behav Brain Res 74:189-197.

de Brabander JM, de Bruin JP, van Eden CG (1991) Comparison of the effects of neonatal and adult medial prefrontal cortex lesions on food hoarding and spatial delayed alternation. Behav Brain Res 42:67-75.

Dias R, Aggleton JP (2000) Effects of selective excitotoxic prefrontal lesions on acquisition of nonmatching- and matching-to-place in the T-maze in the rat: differential involvement of the prelimbic-infralimbic and anterior cingulate cortices in providing behavioural flexibility. Eur J Neurosci 12:4457-4466.

Elliott R, Dolan RJ (1998) Activation of different anterior cingulate foci in association with hypothesis testing and response selection. NeuroImage 8:17-29.

Feenstra MG, Botterblom MH (1996) Rapid sampling of extracellular dopamine in the rat prefrontal cortex during food consumption, handling and exposure to novelty. Brain Res 742:17-24.

Fried PA (1972) Septum and behavior: a review. Psychol Bull 78:292-310. Gehring WJ, Willoughby AR (2002) The medial frontal cortex and the rapid processing of monetary gains and losses. Science 295:2279-2282.

Groenewegen HJ, Berendse HW, Wolters JG, Lohman AH (1990) The anatomical relationship of the prefrontal cortex with the striatopallidal system, the thalamus and the amygdala: evidence for a parallel organization. Prog Brain Res 85:95-116.

Haber SN, Kunishio K, Mizobuchi M, Lynd-Balta E (1995) The orbital and medial prefrontal circuit through the primate basal ganglia. J Neurosci 15:4851-4867.

Howell DC (1997) Statistical methods for psychology, Ed 4. Belmont, CA: Duxbury.

Jackson ME, Moghaddam B (2001) Amygdala regulation of nucleus accumbens dopamine output is governed by the prefrontal cortex. J Neurosci 21:676-681.

Jarrard LE (1989) On the use of ibotenic acid to lesion selectively different components of the hippocampal formation. J Neurosci Methods 29:251-259.

Jedema HP, Moghaddam B (1996) Characterization of excitatory amino acid modulation of dopamine release in the prefrontal cortex of conscious rats. J Neurochem 66:1448-1453.

Jung MW, Qin Y, McNaughton BL, Barnes CA (1998) Firing characteristics of deep layer neurons in prefrontal cortex in rats performing spatial working memory tasks. Cereb Cortex 8:437-450.

Knutson B, Adams CM, Fong GW, Hommer D (2001) Anticipation of increasing monetary reward selectively recruits nucleus accumbens. J Neurosci 21:RC159(1-5).

Kolb B (1984) Functions of the frontal cortex of the rat: a comparative review. Brain Res 320:65-98.

Lacroix L, Broersen LM, Weiner I, Feldon J (1998) The effects of excitotoxic lesion of the medial prefrontal cortex on latent inhibition, prepulse inhibition, food hoarding, elevated plus maze, active avoidance and locomotor activity in the rat. Neuroscience 84:431-442.

Mogenson GJ, Jones DL, Yim CY (1980) From motivation to action: functional interface between the limbic system and the motor system. Prog Neurobiol 14:69-97.

Murase S, Grenhoff J, Chouvet G, Gonon FG, Svensson TH (1993) Prefrontal cortex regulates burst firing and transmitter release in rat mesolimbic dopamine neurons studied in vivo. Neurosci Lett 157:53-56.

Neill DB, Justice JB (1981) An hypothesis for a behavioral function of dopaminergic transmission in nucleus accumbens. In: The neurobiology of the nucleus accumbens (Chronister RB, Defrance JF, eds). Brunswick, Canada: Hue Institute.

O'Doherty J, Kringelbach ML, Rolls ET, Hornak J, Andrews C (2001) Abstract reward and punishment representations in the human orbitofrontal cortex. Nat Neurosci 4:95-102.

Paus T (2001) Primate anterior cingulate cortex: where motor control, drive and cognition interface. Nat Rev Neurosci 2:417-424.

Paxinos G, Watson C (1998) The rat brain in stereotaxic coordinates, Ed 4. San Diego: Academic.

Pratt WE, Mizumori SJ (2001) Neurons in rat medial prefrontal cortex show anticipatory rate changes to predictable differential rewards in a spatial memory task. Behav Brain Res 123:165-183.

Preuss TM (1995) Do rats have prefrontal cortex? The Rose-WoolseyAkert program reconsidered. J Cognit Neurosci 7:1-24.

Ragozzino ME, Detrick S, Kesner RP (1999) Involvement of the prelimbic-infralimbic areas of the rodent prefrontal cortex in behavioral flexibility for place and response learning. J Neurosci 19:4585-4594.

Richardson NR, Gratton A (1998) Changes in medial prefrontal cortical dopamine levels associated with response-contingent food reward: an electrochemical study in rat. J Neurosci 18:9130-9138.

Rogers RD, Owen AM, Middleton HC, Williams EJ, Pickard JD, Sahakian BJ, Robbins TW (1999) Choosing between small, likely rewards and large, unlikely rewards activates inferior and orbital prefrontal cortex. J Neurosci 19.9029-9038.

Salamone JD, Cousins MS, Bucher S (1994) Anhedonia or anergia? Effects of haloperidol and nucleus accumbens dopamine depletion on instrumental response selection in a T-maze cost/benefit procedure. Behav Brain Res 65:221-229.

Salamone JD, Cousins MS, Snyder BJ (1997) Behavioral functions of nucleus accumbens dopamine: empirical and conceptual problems with the anhedonia hypothesis. Neurosci Biobehav Rev 21:341-359.

Seamans JK, Floresco SB, Phillips AG (1995) Functional differences between the prelimbic and anterior cingulate regions of the rat prefrontal cortex. Behav Neurosci 109:1063-1073.

Sesack SR, Deutch AY, Roth RH, Bunney BS (1989) Topographical organization of the efferent projections of the medial prefrontal cortex in the rat: an anterograde tract-tracing study with Phaseolus vulgaris leucoagglutinin. J Comp Neurol 290:213-242.

Taber MT, Fibiger HC (1995) Electrical stimulation of the prefrontal cortex increases dopamine release in the nucleus accumbens of the rat: modulation by metabotropic glutamate receptors. J Neurosci 15:3896-3904

Uylings HB, van Eden CG (1990) Qualitative and quantitative comparison of the prefrontal cortex in rat and in primates, including humans. Prog Brain Res 85:31-62. 